\title{
Bimodal Antibody-Titer Decline Following BNT162b2 Mrna Anti-SARS-Cov-2 Vaccination In Healthcare Workers of The INT - IRCCS "Fondazione Pascale" Cancer Center (Naples, Italy)
}

Maria Antonietta Isgrò

Istituto Nazionale Tumori - IRCCS Fondazione Pascale

Giusy Trillò

Istituto Nazionale Tumori - IRCCS Fondazione Pascale

Luigi Russo

Istituto Nazionale Tumori - IRCCS Fondazione Pascale

Anna Lucia Tornesello

Istituto Nazionale Tumori - IRCCS Fondazione Pascale

Luigi Buonaguro

Istituto Nazionale Tumori - IRCCS Fondazione Pascale

Maria Lina Tornesello

Istituto Nazionale Tumori - IRCCS Fondazione Pascale

Leonardo Miscio

Istituto Nazionale Tumori - IRCCS Fondazione Pascale

Nicola Normanno

Istituto Nazionale Tumori - IRCCS Fondazione Pascale

Attilio Antonio Montano Bianchi

Istituto Nazionale Tumori - IRCCS Fondazione Pascale

Franco Maria Buonaguro

Istituto Nazionale Tumori - IRCCS Fondazione Pascale

Ernesta Cavalcanti ( $\nabla$ e.cavalcanti@istitutotumori.na.it)

Istituto Nazionale Tumori - IRCCS Fondazione Pascale

\section{Research Article}

Keywords: Bimodal titer, BNT162b2 (Pfizer-BioNTech), antibody avidity, immunoprotective titer

Posted Date: February 8th, 2022

DOI: https://doi.org/10.21203/rs.3.rs-1314541/v1 
License: (c) (i) This work is licensed under a Creative Commons Attribution 4.0 International License. Read Full License 


\section{Abstract}

Background: Both SARS-CoV-2 mRNA-based vaccines [BNT162b2 (Pfizer-BioNTech) and mRNA-1273 (Moderna)] have shown high efficacy, with very modest side effects in limiting transmission of SARSCoV-2 and in preventing the severe COVID-19 disease, characterized by a worrying high occupation of intensive care units (ICU), high frequency of intubation and ultimately high mortality rate. At the INT, in Naples, only the BNT162b2 / Pfizer vaccine has been administered to cancer patients and healthcare professionals aged 16 and over.

In the present study, the antibody response levels and their decline were monitored in an interval of 6-9 months after vaccine administration in the two different cohorts of workers of the INT - IRCCS "Fondazione Pascale" Cancer Center (Naples, Italy): the group of individuals previously infected with SARS-CoV-2 and vaccinated with a single dose; and that of individuals negative for previous exposure to SARS-CoV-2 vaccinated with two doses 21 days apart.

Methods: Specific anti-RBD (receptor-binding domain) titers against trimeric spike glycoprotein (S) of SARS-CoV-2 by Roche Elecsys Anti-SARS-CoV-2 S ECLIA immunoassay were determined in serum samples of 27 healthcare workers with a previously documented history of SARS-CoV-2 infection and 123 healthcare workers without, during antibody titers' monitoring. Moreover, geometric mean titers (GMT) and relative fold changes (FC) were calculated.

Results: Bimodal titer decline was observed in both previously infected and uninfected SARS-CoV-2 subjects. A first rapid decline was followed by a progressive slow decline in the $6 / 9$ month-period before the further vaccine boost. The trend was explained by 2 different mathematical models, exponential and power function, the latter revealing as predictive of antibody titer decline either in infected or in not previously infected ones. The value of the prolonged lower vaccine titer was about 1 log below in the 6/9month interval after the single dose for previously infected individuals with SARS-CoV-2 and the two doses for those not previously infected. The titer change, after the boost dose administration, on the other hand, was $\geq 1.5 \mathrm{FC}$ higher than the titers at the 6/9-month time-points in both cohorts. A similar quantitative immune titer was observed in both cohorts 8 days after the last boost dose. The subsequent immunoresponse trend remains to be verified.

Discussion: The results show that a very rapid first decline, from the highest antibody peak, was followed by a very slow decline which ensured immune protection lasting more than six months. The apparent absence of adverse effects of the rapid decline on the vaccine's immune protective role has been related to a large majority of low avidity antibodies induced by current vaccines. High avidity antibodies with prolonged anti-transmission efficacy show a longer half-life and are lost over a longer interval period. The cellular immunity, capable of preventing severe clinical diseases, lasts much longer. The unbalanced dual activity (cellular vs humoral) while effective in limiting ICU pressure and overall mortality, does not protect against transmission of SARS-CoV-2, resulting in high circulation of the virus among unvaccinated subjects, including the younger population, and the continuous production of variants characterized by changes in transmissibility and pathogenicity. The high mutation rate, peculiar to the RNA virus, can 
however lead to a dual opposite results: selection of defective and less efficient viruses up to extinction; risk of more efficiently transmitted variants as the current omicron pandemic.

Conclusions: In conclusion the current bimodal antibody-titer decline, following BNT162b2 mRNA antiSARS-CoV-2 vaccination, needs a further extended analysis to verify the protective borderline levels of immunity and the optimal administration schedule of vaccine boosters. Our current results can contribute to such goal, besides a direct comparison of other FDA-approved and candidate vaccines.

\section{Background}

Severe Acute Respiratory Syndrome Coronavirus-2 (SARS-CoV-2), a positive-sense single-stranded RNA virus belonging to the Coronaviridae family, causes coronavirus disease 2019 (COVID-19), a respiratory syndrome evolving frequently to severe pneumonia, respiratory and multi-visceral failure and causing death in some patients with comorbidity [1].

Preventive protection against many infectious diseases is mediated by a functional, persistent antibody response, which is therefore a critical immune correlate for many licensed human vaccines. The durability of vaccine-acquired antibody responses varies greatly among antigens [2]. Antibodies induced by viral infections, or by vaccination with live-attenuated viruses, can persist for decades. However, most vaccines based on protein antigens require repeated immunizations to generate immunological memory, and to maintain antibody responses above protective levels [3]. The level of antigen-antibody binding avidity, a qualitative response index, can also correlate with protection. Nevertheless, low-avidity antibodies have been associated with antibody-mediated disease enhancement following pandemic influenza vaccinations [4].

Moreover, inadequate levels of avidity maturation (the latter defined as the increase of avidity over time) can heighten susceptibility to viral infection [5]. Thus, both quantitative and qualitative yardsticks can determine vaccine efficacy. Vaccine adjuvants are linked to both of these aspects of the antibody response. By enhancing innate immunity, they promote activation of naive $B$ cells and CD4+ $T$ cells [6]. Antibody avidity (as well as titers) can also be significantly enhanced by repeated immunization, particularly when the vaccine contains an effective adjuvant [7].

The immune system represents an important component against the viral infection by the titer and avidity of neutralizing antibodies production. The trimeric spike glycoprotein (S) of SARS-CoV-2 is a key target for virus neutralizing antibodies and the prime candidate for vaccine development [8]. The protein $S$ binds its cellular receptor on the host cells, human angiotensin converting enzyme 2 (ACE2), through a receptor-binding domain (RBD) [9].

Both SARS-CoV-2 mRNA-based vaccines containing the messenger RNA that encodes the SARS-CoV-2 S in small lipid particles [BNT162b2 (Pfizer-BioNTech) and mRNA-1273 (Moderna)] have shown high efficacy, with very modest side effects in limiting transmission of SARS-CoV-2 and in preventing the 
severe COVID-19 disease, characterized by a worrying high occupation of intensive care units (ICU), high frequency of intubation and ultimately high mortality rate.

On $22^{\text {nd }}$ December 2020, the Italian regulatory agency for drugs AIFA (Agenzia Italiana del Farmaco) authorized in Italy the use of BNT162b2/Pfizer vaccine, in 2 doses with an interval of 21 days between the doses [10]. Recent studies have found that subjects infected with COVID-19 develop higher antibody titers after vaccination [11] and present protective immunity for at least 6 months [12], but the impact of previous exposure to SARS-CoV-2 on immune response elicited by the vaccines needs to be further verified in larger trial studies. Several studies have shown that the immune response to the vaccine after the first dose is substantially more pronounced in individuals with pre-existing immunity and it is similar to the immune response developed after the second dose in individuals not previously infected $[11,13$, $14,15]$. However, few data are available on the accurate monitoring of the titers' decline, response to further boosters, optimal vaccine dosage and role of different adjuvants on vaccine efficacy.

In the present study, the antibody response levels and their decline were monitored in an interval of $6 / 9$ months after vaccine administration in the two different cohorts of workers of the INT - IRCCS "Fondazione Pascale" Cancer Center (Naples, Italy), established since 2020 [16]: the group of individuals previously infected with SARS-CoV-2 and vaccinated with a single dose; and that of individuals negative for previous exposure to SARS-CoV-2 vaccinated with two doses 21 days apart.

\section{Materials And Methods}

\section{Sample size}

According to our national recommendations, the internal health surveillance program for healthcare workers implemented the following BNT162b2/Pfizer vaccination schedule: 1 dose was administered to subjects previously infected to SARS-CoV-2, and 2 doses (with an interval of 21 days) to subjects not infected. Most of healthcare providers underwent also boost dose. The program contemplated the evaluation of antibody responses by determining anti-RBD titers at three times: the day before vaccination (baseline anti-S antibody titer), 20 days after the first dose and 8 days after the second dose for not previously infected workers and 8 days after the unique dose for previously infected healthcare providers. Further data were collected by determining antibody titers 1 month after the preliminary completion of vaccination cycle and 8 days after the boost dose administration for both cohorts. Time points were defined as follows:

- for not infected subjects:

- T0 = baseline pre-vaccination

- T1 $=20$ days after the first dose

- T2 = 8 days after the second dose, distinguishing: 
- T2 I = 8 days after the second dose

- T2 II = 1 month after the second dose

- T3 = 3 months after the second dose

- T6 = 6 months after the second dose

- T9 = 9 months after the second dose

- T boost $=8$ days after the administration of a further boost dose

- for previously infected subjects:

- T0 = baseline pre-vaccination after SARS-CoV-2 infection

- $\mathrm{T} 1$ = 8 days after the unique dose

- T2 = 1 month after the unique dose

- T3 = 3 months after the unique dose

- T6 = 6 months after the unique dose

- T9 = 9 months after the unique dose

- T boost $=8$ days after the administration of a further boost dose .

Data regarding 150 healthcare workers of INT - IRCCS "Fondazione Pascale" Cancer Centre (27 and 123 with history/no history of COVID-19 infection, defined as infected and not infected subjects, respectively) were analyzed. Moreover, in order to exactly quantify and extend the analysis to a 9-month interval two subgroups were extrapolated for the previously COVID-infected $(n=3)$ and not infected subjects $(n=20)$. The study was performed under the statements of Declaration of Helsinki and approved by the local Institutional Ethics Committee. All participants gave their informed consent.

\section{Assay}

Roche Elecsys Anti-SARS-CoV-2 S electrochemiluminescence immunoassay (ECLIA) for the in vitro quantitative determination of antibodies (including $\mathrm{Ig}$ ) against spike RBD of SARS-CoV-2 in human serum was performed on Roche Cobas e 801 module. According to the manufacturer, the correlation test between Roche Elecsys Anti - SARS-CoV-2 S units per $\mathrm{mL}$ and WHO International Standards for antiSARS-CoV-2 immunoglobulins showed an excellent correlation $\left(r^{2}=0.9992\right.$, slope $=0.972$, intercept $=$ 0.0072), thus allowing to consider specific Roche Elecsys Anti-SARS-CoV-2 S U/mL units equivalent to WHO International Standard BAU/mL (Binding Arbitrary Units per $\mathrm{mL}$ ). Measuring range spanned from $0.4 \mathrm{BAU} / \mathrm{mL}$ to $250.0 \mathrm{BAU} / \mathrm{mL}$, requiring a 1:10 dilution for samples with concentrations $>250.0 \mathrm{BAU} / \mathrm{mL}$, 
extending the measuring range until to $2500.0 \mathrm{BAU} / \mathrm{mL}$; values higher than $0.8 \mathrm{BAU} / \mathrm{mL}$ were considered positive. Serum samples with antibody titers $>2500.0 \mathrm{BAU} / \mathrm{mL}$, from the selected subgroups above specified, were further diluted in order to obtain the exact quantitative concentration.

\section{Statistical analysis}

Statistical analysis was performed by using the Statistical Package for Social Science (SPSS Inc., Chicago, IL, USA), version 27.0. Distribution of variables was evaluated by Shapiro-Wilk test; parametric data were represented as mean \pm standard deviation (SD), whilst non-parametric variables were expressed as median (IR - Interquartile Range). Two-tailed Mann-Whitney for independent variables test was used to compare groups. Values lower than $0.4 \mathrm{BAU} / \mathrm{mL}$ were assumed as 0.4 and values higher than $2500.0 \mathrm{BAU} / \mathrm{mL}$ were reported as $2500.0 ; p$ values $<0.05$ were considered statistically significant. Moreover, geometric mean titers (GMT) and relative fold changes (FC) were calculated. To overcome the novelty of general bead-based linear models, used to evaluate post-to-pre vaccination antibody titer increase or subsequent titer decline, the used statistic methods were extrapolated from Zaccaro et al. [17]. Different mathematical models were tested and evaluated by fitting median values extrapolated by immunoassays at different time-points for previously and not previously infected healthcare providers, in order to study and explain the antibody decline kinetics.

\section{Results}

The enrolled cohort of healthcare workers (27 previously infected and 123 not infected) was represented as follows: 27 seropositive cases, 17 female and 10 male subjects with an overall mean age of 49.1 years (SD \pm 8.9 , range $34-65$ ); and 123 seronegative cases, 80 female and 43 male subjects with an overall mean age of 46.2 years (SD \pm 11.4 , range $23-67$ ) (Table 1 ).

Data regarding monitoring during the interval from baseline to boost dose administration are showed in Table 2 and Figure 1. At each time-point, two-tailed Mann-Whitney $U$ test showed significantly higher antibody titer concentrations in infected subjects in comparison with not previously infected ones, except for $T$ boost at which previously infected workers presented higher (but not statistically significant) levels of antibodies.

Despite the upper limit of $2500.0 \mathrm{BAU} / \mathrm{mL}$ imposed by the Roche method, a bimodal titer decline, before the further boost dose administration, was observed in most subjects. This finding was confirmed on data obtained from further diluted samples. In order to better define antibody titers' trend in the interval immediately after the administration of the unique dose or the second dose, the T2 for previously infected subjects, and T2 I - T2 II time-points for not infected ones, respectively, were included (Table 3, Figures 2a$2 b)$. Such analysis allowed to show for not previously infected workers a first rapid decline in the interval T2 I - T2 II with a negative slope of -2495.5 followed by a progressive slow decline in the $6 / 9$ monthperiod before the further vaccine boost (slope of -847.3 in the interval T2 II - T3, -342.2 in the interval T3 T6, -123.1 in the interval T6 - T9), with a slope of -3342.9 in the interval T2 I - T3 and an overall negative slope of -3808.2 from T2 I to T9 (Table 3, Figure 2a). Peculiarly, in the cohort of previously infected 
workers, after an unexpected and unreported initial median increase of antibody titer in the T1 - T2 interval (positive slope of 2550), a rapid decline followed by a slower decrease was observed (Table 3 ), with a negative slope of -7562 in the interval T2 - T3, -1009 (T3 - T6), -582 (T6 - T9) and an overall negative slope of -9153 reported from T2 to T9 (Table 3, Figure 2b). Both in previously infected cohort and in not previously infected one, 2 different mathematical models were analyzed to study and calculate the antibody decline kinetics: an exponential $v s$. a power function model.

In not previously infected workers the following equations were detected:

$y=5982.8 e^{-0.546 x}($ exponential)

and

$y=4407.4 x^{-1.392}$ (power)

for exponential and power function models, with $R^{2}=0.9502$ and 0.9971 , respectively (Figures 3a-3b).

In previously infected subjects the following equations were detected:

$y=16028 e^{-0.716 x}($ exponential)

and

$y=9533.8 x^{-1.599}$ (power)

for exponential and power function models, with $R^{2}=0.9136$ and 0.988 , respectively (Figures $4 \mathrm{a}-4 \mathrm{~b}$ ).

By calculating GMT at each time-point and relative FC, it was found that the prolonged lower vaccine titer was about 1 log below in the 6/9-month interval after the single dose for previously infected individuals with SARS-CoV-2 and the two doses for those not previously infected (Table 4). The mean change in titer after the boost dose administration, on the other hand, was equal to or more than $1.5 \mathrm{FC}$ higher than the T6 - T9 time-points in both cohorts (1.5 and 1.6 higher than T6 and T9 for previously infected workers, and 1.7 and 1.8 higher than T6 and T9 for not previously infected ones, respectively) (Table 4). A mean increase of 0.7 and 0.9 FC was found comparing T boost - T2 time-points in infected subjects and in not previously infected ones, respectively (Table 4). At $\mathrm{T}$ boost time-point, previously infected workers presented higher (but not statistically significant) levels of antibodies in respect to not previously infected cohort (Table 4, Figures 5a - 5b). At T boost time-point, however, a similar anti-S antibody titer was observed in previously infected and not-infected subjects suggesting that the vaccination boost was able to induce an equivalent immune response among the two groups eliminating any difference related to the previous infection.

\section{Discussion}


In accordance with first data reported in literature [11, 13, 18], our findings confirm that administration of a single dose to previously infected subjects is sufficient to elicit an adequate immunoresponse, the latter resulting more sustained in terms of concentration and duration if compared to antibody response of not previously infected cohort. Nevertheless, for the first time in literature, we demonstrated a bimodal decline kinetic characterized by a very rapid first decline from the highest antibody peak ( 1 month after the unique vaccine dose in previously infected workers and 8 days after the second dose in not previously infected one), followed by a slow decline which ensures a long-term immune protection lasting more than six months. Our data fit a power function decline curve, which allows the calculation and identification of a specific trend that can predict the rate of decline in antibody titer with excellent accuracy in both previously infected and uninfected individuals. Moreover, the initial more pronounced decline observed in previously infected subjects (demonstrated by higher negative slopes) was counterbalanced by the less robust response to the additional boost dose, resulting in overlapping of antibody titers in the 2 different cohorts 8 days after boost vaccine administration (T boost).

The apparent absence of negative effects related to the rapid decline on the vaccine's immune protective role has been referred to a large majority of low avidity antibodies induced in the early post-vaccination stages $[19,20,21]$. High avidity antibodies with prolonged anti-transmission efficacy show a longer halflife and are lost over a longer interval period. Protective humoral immune response is generally characterized by a parabolic trend with a protective plateau, several months long, following natural infection as well as vaccine induced active immunity, also for the latest developed subunits vaccines. As paradigm the long-lasting immune response following a single dose of L1-based Virus-like particles (VLPs), the anti- DNA papillomavirus (HPV) vaccine [22], and following multiple doses of a recombinant E2-based vaccine against the RNA-hepatitis E virus (HEV) [23] can be considered. The trend observed in the current anti-SARS-CoV-2 vaccination shows, instead, after a rapid increment an asymptotic hyperbolic trend towards a modest not-protective immune response gradually declining below protective immune threshold. The high titer stage is lost in less than 30 days and is referred mainly to low-avidity antibodies, leaving the protective activity to the low-titer stage ranging from 1893.4 to $469.5 \mathrm{BAU} / \mathrm{ml}$ for the subject without previous COVID infection and from 2806.4 to $1152.2 \mathrm{BAU} / \mathrm{ml}$ for those with a previous COVID infection (GMT from Figures $2 a$ and $2 b$, respectively). The alternative dramatic inference would be that the protective period is just a month.

The identification and quantification of the protective lower antibody level, whose avidity along with neutralizing activity increases over time [24], becomes relevant for monitoring the individual protection level and selecting the appropriate further boosting time. Moreover, it becomes a reference value to compare the efficacy of other vaccines as well as their delivery and formulation (including adjuvants) besides the possibility to develop pre-vaccination strategies to improve the immune response to vaccines [25].

Fortunately, the cellular immunity, capable of preventing severe clinical diseases, seems to last much longer. The complementary immune response (short-lived humoral immunity $v s$ long-lasting cellular immunity), although effective in limiting overall mortality, with the consequent reduction of pressure in 
the ICU, does not prevent and does not protect against transmission of SARS-CoV-2, resulting in high circulation of the virus among unvaccinated subjects, including the younger population, and the continuous production of variants characterized by changes in transmissibility and pathogenicity. The high mutation rate, peculiar to the RNA virus, can however lead to a dual opposite result: selection of defective and less efficient viruses up to extinction [26, 27]; risk of more efficiently transmitted variants as the current omicron pandemic [28, 29].

\section{Conclusions}

In conclusion, further extended analyses are mandatory to confirm our preliminary findings in larger cohorts of subjects (including patients and immunocompromised individuals), in order to monitor antibody titers also after the administration of further boost doses, verify the protective borderline levels of immunity and define the optimal administration schedule of vaccine boosters. Our results, highlighting the current bimodal antibody-titer decline after BNT162b2 mRNA anti-SARS-CoV-2 vaccination, can contribute to such goal, besides a direct comparison of other FDA-approved and candidate vaccines.

\section{Abbreviations}

ACE2: human Angiotensin Converting Enzyme 2

AIFA: Agenzia Italiana del Farmaco, the Italian regulatory agency for drugs

$\mathrm{BAU} / \mathrm{mL}$ : Binding Arbitrary Units per $\mathrm{mL}$

COVID-19: Coronavirus disease 2019

ECLIA: Electrochemiluminescence Immunoassay

FC: Fold Change

GMT: Geometric Mean Titer

HPV: Human papilloma virus

HEV: Hepatitis E Virus

ICU: Intensive Care Units

IR: Interquartile Range

RBD: Receptor-Binding Domain

S: trimeric spike glycoprotein

SARS-CoV-2: Severe Acute Respiratory Syndrome caused by Coronavirus 2 
Tx Time Points:

For not infected subjects: $\mathrm{T} 0$ = baseline pre-vaccination, $\mathrm{T} 1=20$ days after the first dose, $\mathrm{T} 2=8$ days after the second dose, distinguishing T2 I = 8 days after the second dose and T2 II = 1 month after the second dose, $\mathrm{T} 3=3$ months after the second dose, $\mathrm{T} 6=6$ months after the second dose, $\mathrm{T} 9=9$ months after the second dose, $\mathrm{T}$ boost $=8$ days after the administration of a further boost dose

For previously infected subjects: T0 $=$ baseline pre-vaccination after SARS-CoV-2 infection, $\mathrm{T} 1=8$ days after the unique dose, T2 = 1 month after the unique dose, T3 = 3 months after the unique dose, T6 = 6 months after the unique dose, $\mathrm{T} 9=9$ months after the unique dose, $\mathrm{T}$ boost $=8$ days after the administration of a further boost dose.

VLP: Virus-like particles

\section{Declarations}

Ethics approval and consent to participate: The Institutional Ethics Committee approved the study, performed under the statements of Declaration of Helsinki, document \#34/21 "RUI-SARS" (16/06/21).

Consent for publication: All authors gave their consent for publication.

Availability of data and materials: All relevant data and their evaluation are reported in the manuscript.

Competing interests: All authors declared to have no conflict of interest.

Funding: Ricerca Corrente Ministero Salute 2021 - Progetto "Caratterizzazione Bio - Molecolare Del Virus SARS-CoV-2 E Dei Cofattori Dell'infiammazione Implicati Nella Patogenesi Della Covid-19" programma anti COVID-19 della Regione Campania Anno 2020 dal titolo "Misure Urgenti in materia di contenimento e gestione della emergenza epidemiologica da COVID-19 DRG N.140/2020".

Acknowledgements: The authors thank all the health workers of the INT - IRCCS "Fondazione Pascale" involved in the COVID monitoring and those of the Laboratory Medicine for their valuable contribution.

\section{References}

1. Zhou F, Yu T, Du R, Fan G, Liu Y, Liu Z, et al. Clinical course and risk factors for mortality of adult inpatients with COVID-19 in Wuhan, China: a retrospective cohort study. Lancet. 2020 Mar 28;395(10229):1054-1062. doi: 10.1016/S0140-6736(20)30566-3

2. Hangartner, L., Zinkernagel, R. M. \& Hengartner, H. Antiviral antibody responses: the two extremes of a wide spectrum. Nat Rev Immunol. 2006 Mar;6(3):231-43 doi: 10.1038/nri1783 
3. Antia, A. et al. Heterogeneity and longevity of antibody memory to viruses and vaccines. PLoS Biol. 2018 Aug 10;16(8):e2006601. doi: 10.1371/journal.pbio.2006601

4. Smatti, M. K., Al Thani, A. A. \& Yassine, H. M. Viral-induced enhanced disease illness. Front Microbiol. 2018 Dec 5;9:2991. doi: 10.3389/fmicb.2018.02991

5. Lee, Y. C. et al. Haemophilus influenzae type $b$ vaccine failure in children is associated with inadequate production of high-quality antibody. Clin Infect Dis. 2008 Jan 15;46(2):186-92. doi: $10.1086 / 524668$

6. Del Giudice, G., Rappuoli, R. \& Didierlaurent, A. M. Correlates of adjuvanticity: a review on adjuvants in licensed vaccines. Vaccine. 2015 Aug 7;33(33):4146-54. doi: 10.1016/j.vaccine.2015.05.081

7. Eidem, S. et al. Persistence and avidity maturation of antibodies to $A(H 1 N 1)$ pdm09 in healthcare workers following repeated annual vaccinations. Vaccine. 2015 Aug 7;33(33):4146-54. doi: 10.1016/j.vaccine.2015.05.081

8. Vogel AB, Kanevsky I, Che Y, Swanson KA, Muik A, Vormehr M, et al. BNT162b vaccines protect rhesus macaques from SARS-CoV-2. Nature. 2021 Apr;592(7853):283-289. doi: 10.1038/s41586-02103275-y

9. Zhou P, Yang XL, Wang XG, Hu B, Zhang L, Zhang W, et al. A pneumonia outbreak associated with a new coronavirus of probable bat origin. Nature. 2020 Mar;579(7798):270-273. doi: 10.1038/s41586020-2012-7

10. Filia A, Rota MR, D'Ancona FP. Comirnaty (BNT162b2), Il primo vaccino contro il COVID-19 approvato in Europa e in Italia: Istituto Superiore di Sanità -Dipartimentomalattieinfettive;2021. https://www.epicentro.iss.it/vaccini/covid-19-vaccino-pfizer-biontech

11. Cavalcanti E , Isgrò MA, Rea D, Di Capua L, Trillò G, Russo L, et al. Vaccination strategy and anti SARS-CoV-2 S titers in healthcare workers of the INT - IRCCS "Fondazione Pascale" Cancer Center (Naples, Italy). Infect Agent Cancer. 2021 May 12;16(1):32. doi: 10.1186/s13027-021-00375-2

12. Dan JM, Mateus J, Kato Y, Hastie KM, Yu ED, Faliti CE, et al. Immunological memory to SARS-CoV-2 assessed for up to 8 months after infection. Science. 2021 Feb 5;371(6529):eabf4063. doi: $10.1126 /$ science.abf 4063

13. Krammer F, Srivastava K, Alshammary H, Amoako AA, Awawda MH, et al. Antibody Responses in Seropositive Persons after a Single Dose of SARS-CoV-2 mRNA Vaccine. N Engl J Med. 2021 Apr 8;384(14):1372-1374. doi: 10.1056/NEJMc2101667

14. Padoan A, Dall'Olmo L, Rocca FD, Barbaro F, Cosma C, Basso D, et al. Antibody response to first and second dose of BNT162b2 in a cohort of characterized healthcare workers. Clin Chim Acta. 2021 Aug;519:60-63. doi: 10.1016/j.cca.2021.04.006

15. Blain H, Tuaillon E, Gamon L, Pisoni A, Miot S, Picot MC, et al. Spike antibody levels of nursing home residents with or without prior COVID-19 3 weeks after a single BNT162b2 vaccine dose. JAMA. 2021 Apr 15;325(18):1898-1899. doi: 10.1001/jama.2021.6042

16. Buonaguro, F.M., Botti, G., Ascierto, P.A. et al. The clinical and translational research activities at the INT - IRCCS "Fondazione Pascale" cancer center (Naples, Italy) during the COVID-19 
pandemic. Infect Agents Cancer15, 69 (2020). https://doi.org/10.1186/s13027-020-00330-7

17. Zaccaro DJ, Wagener DK, Whisnant CC, Staats HF. Evaluation of vaccineinduced antibody responses: impact of new technologies. Vaccine. 2013 Jun 7;31(25):2756-61. doi: 10.1016/j.vaccine.2013.03.065

18. Levi R, Azzolini E, Pozzi C, Ubaldi L, Lagioia M, Mantovani A, Rescigno M. One dose of SARS-CoV-2 vaccine exponentially increases antibodies in individuals who have recovered from symptomatic COVID-19. J Clin Invest. 2021 Jun 15;131(12):e149154. doi: 10.1172/JCI149154. PMID: 33956667; PMCID: PMC8203458.

19. Budroni S, Buricchi F, Cavallone A, Bourguignon P, Caubet M, Dewar V, D'Oro U, Finco O, Garçon N, El Idrissi M, Janssens M, Leroux-Roels G, Marchant A, Schwarz T, Van Damme P, Volpini G, van der Most R, Didierlaurent AM, Burny W. Antibody avidity, persistence, and response to antigen recall: comparison of vaccine adjuvants. NPJ Vaccines. 2021 May 21;6(1):78. doi: 10.1038/s41541-02100337-0. PMID: 34021167; PMCID: PMC8140094.

20. Tretyn A, Szczepanek J, Skorupa M, Jarkiewicz-Tretyn J, Sandomierz D, Dejewska J, Ciechanowska K, Jarkiewicz-Tretyn A, Koper W, Pałgan K. Differences in the Concentration of Anti-SARS-CoV-2 IgG Antibodies Post-COVID-19 Recovery or Post-Vaccination. Cells. 2021;10(8):1952. https://doi.org/10.3390/cells10081952

21. Levin EG, Lustig Y, Cohen C, Fluss R, Indenbaum V, Amit S, et al. Waning Immune Humoral Response to BNT162b2 Covid-19 Vaccine over 6 Months. The New England journal of medicine. 2021. Epub 2021/10/07. pmid:34614326

22. Harper DM, DeMars LR. HPV vaccines - a review of the first decade. Gynecol Oncol. 2017;146(1):196-204. doi:10.1016/j.ygyno.2017.04.004.

23. Wen, GP., He, L., Tang, ZM. et al. Quantitative evaluation of protective antibody response induced by hepatitis E vaccine in humans. Nat Commun11, 3971 (2020). https://doi.org/10.1038/s41467-02017737-w

24. Scheiblauer $H$, Nübling CM, Wolf T, Khodamoradi Y, Bellinghausen $C$, Sonntagbauer M, Esser-Nobis $K$, Filomena A, Mahler V, Maier TJ, Stephan C. Antibody response to SARS-CoV-2 for more than one year - kinetics and persistence of detection are predominantly determined by avidity progression and test design. J Clin Virol. 2022 Jan;146:105052. doi: 10.1016/j.jcv.2021.105052. Epub 2021 Dec 4. PMID: 34920374; PMCID: PMC8642248.

25. Tsang JS, Dobaño C, VanDamme P, Moncunill G, Marchant A, Othman RB, Sadarangani M, Koff WC, Kollmann TR. Improving Vaccine-Induced Immunity: Can Baseline Predict Outcome? Trends Immunol. 2020 Jun;41(6):457-465. doi: 10.1016/j.it.2020.04.001. Epub 2020 Apr 8. PMID: 32340868; PMCID: PMC7142696.

26. Jogalekar MP, Veerabathini A, Gangadaran P. SARS-CoV-2 variants: A double-edged sword? Exp Biol Med (Maywood). 2021 Aug;246(15):1721-1726. doi: 10.1177/15353702211014146

27. Schiøler H, Knudsen T, Brøndum RF, Stoustrup J, Bøgsted M. Mathematical modelling of SARS-CoV-2 variant outbreaks reveals their probability of extinction. Sci Rep. 2021 Dec 30;11(1):24498. doi: 
10.1038/s41598-021-04108-8. PMID: 34969959; PMCID: PMC8718533

28. Chaudhry MZ, Eschke K, Hoffmann M, Grashoff M, Abassi L, Kim Y, Brunotte L, Ludwig S, Kröger A, Klawonn F, PöhImann SH, Cicin-Sain L. Rapid SARS-CoV-2 Adaptation to Available Cellular Proteases. J Virol. 2022 Jan 12:jvi0218621. doi: 10.1128/jvi.02186-21. Epub ahead of print. PMID: 35019723

29. Gerdol M, Dishnica K, Giorgetti A. Emergence of a recurrent insertion in the N-terminal domain of the SARS-CoV-2 spike glycoprotein. Virus Res. 2022 Jan 9;310:198674. doi:

10.1016/j.virusres.2022.198674. Epub ahead of print. PMID: 35021068; PMCID: PMC8743576.

\section{Tables}

Table 1 Demographic data of previously infected and not previously infected cohorts of healthcare providers

\section{Male (age) Female (age) Whole cohort (age)}

\begin{tabular}{llll}
\hline & $\mathrm{n}($ mean \pm SD $)$ & $\mathrm{n}($ mean \pm SD $)$ & $\mathrm{n}($ mean \pm SD $)$ \\
\hline Previously infected subjects & $10(49.2 \pm 8.9)$ & $17(49.3 \pm 9.4)$ & $27(49.1 \pm 8.9)$
\end{tabular}

Not previously infected subjects $\quad 43(46.4 \pm 11.4) \quad 80(46.2 \pm 11.3) \quad 123(46.2 \pm 11.4)$

Whole cohort

$53(46.9 \pm 11.0) \quad 97(46.7 \pm 11.1) \quad 150(46.7 \pm 11.0)$

Previously infected subjects $=$ subjects previously infected with SARS-CoV-2

Not previously infected subjects $=$ subjects not infected with SARS-CoV-2

$\mathrm{n}=$ number of subjects

mean $=$ mean age

$\mathrm{SD}=$ standard deviation

Table 2 Vaccine immune response monitoring in previously infected and not previously infected cohorts of healthcare providers 
Median (IR) BAU/mL

\begin{tabular}{llllllll}
\hline T0 & T1 & T2 & T3 & T6 & T9 & T boost & \\
\hline $\begin{array}{l}\text { Previously } \\
\text { infected subjects }\end{array}$ & 35.6 & $>$ & $>2500.0$ & $>2500.0$ & 2098.0 & 1212.5 & $>$ \\
& & 2500.0 & & & & & 2500.0 \\
\cline { 2 - 8 } & $\begin{array}{l}(20.4- \\
91.4)\end{array}$ & & & & $(1565.5->$ & $(957.2-$ & \\
& $\mathrm{n}=27$ & $\mathrm{n}=27$ & $\mathrm{n}=27$ & $\mathrm{n}=27$ & $\mathrm{n}=27$ & $\mathrm{n}=26$ & $\mathrm{n}=5$ \\
\hline & $<0.4$ & 26.5 & $>2500.0$ & 853.4 & 574.0 & 381.0 & $>$ \\
\hline $\begin{array}{l}\text { Not previously } \\
\text { infected subjects }\end{array}$ & & & & & & & 2500.0 \\
& & $(9.4-$ & $(2020.0->$ & $(514.9-$ & $(363.5-$ & $(255.3-$ & \\
& & $68.5)$ & $2500.0)$ & $1252.0)$ & $814.8)$ & $579.4)$ & \\
\cline { 2 - 8 } & $\mathrm{n}=$ & $\mathrm{n}=$ & $\mathrm{n}=123$ & $\mathrm{n}=123$ & $\mathrm{n}=123$ & $\mathrm{n}=62$ & $\mathrm{n}=14$ \\
& 123 & 123 & & & & & \\
\end{tabular}

Previously infected subjects $=$ subjects previously infected with SARS-CoV-2

Not previously infected subjects $=$ subjects not infected with SARS-CoV-2

$\mathrm{n}=$ number of subjects

median $=$ median of anti - SARS-CoV-2 S titers

$\mathrm{IR}=$ Interquartile Range of anti - SARS-CoV-2 S titers

For previously infected subjects: T0 $=$ baseline pre-vaccination after SARS-CoV-2 infection, T1 = 8 days after the unique dose, T2 = 1 month after the unique dose, T3 = 3 months after the unique dose, T6 = 6 months after the unique dose, $\mathrm{T} 9=9$ months after the unique dose, $\mathrm{T}$ boost $=8$ days after the administration of a further boost dose. For not infected subjects: $\mathrm{T} 0=$ baseline pre-vaccination, $\mathrm{T} 1=20$ days after the first dose, T2 = 8 days after the second dose, T3 = 3 months after the second dose, T6 = 6 months after the second dose, $\mathrm{T} 9=9$ months after the second dose, $\mathrm{T}$ boost $=8$ days after the administration of boost dose.

Table 3 Vaccine immune response decline in previously infected and not previously infected cohorts of healthcare providers' diluted samples 
Median (IR) BAU/mL

\begin{tabular}{|c|c|c|c|c|c|c|c|c|}
\hline TO & $\mathrm{T} 1$ & T2 I & T2 II & T3 & T6 & T9 & T boost & \\
\hline \multirow{4}{*}{$\begin{array}{l}\text { Previously } \\
\text { infected } \\
\text { subjects }\end{array}$} & 207.4 & 7706.0 & \multicolumn{2}{|l|}{10256.0} & 2694.0 & 1685.0 & 1103.0 & 52694.5 \\
\hline & $(108.5$ & (7152.0 & \multirow{2}{*}{\multicolumn{2}{|c|}{$(9442.5-11198.5)$}} & $(2661.0$ & $(1623.5$ & (1069.0 & (51223.8 \\
\hline & 244.2) & 9220.5) & & & 2908.0) & $1745.5)$ & 1221.5) & 54165.3) \\
\hline & $n=3$ & $\mathrm{n}=3$ & $n=3$ & & $\mathrm{n}=3$ & $n=3$ & $n=3$ & $n=2$ \\
\hline \multirow{4}{*}{$\begin{array}{l}\text { Not } \\
\text { previously } \\
\text { infected } \\
\text { subjects }\end{array}$} & $<0.4$ & 52.3 & 4290.0 & 1794.5 & 947.2 & 605.0 & 481.8 & 33419.5 \\
\hline & & (30.7- & $(3635.0$ & $(1426.8$ & \multirow{2}{*}{$\begin{array}{l}(671.8- \\
1325.0)\end{array}$} & \multirow{2}{*}{$\begin{array}{l}(410.2- \\
824.6)\end{array}$} & \multirow{2}{*}{$\begin{array}{l}(315.9- \\
625.3)\end{array}$} & (22103.3 \\
\hline & & & $4844.3)$ & 2381.0) & & & & $54130.8)$ \\
\hline & $\mathrm{n}=20$ & $\mathrm{n}=20$ & $\mathrm{n}=20$ & $\mathrm{n}=20$ & $\mathrm{n}=20$ & $\mathrm{n}=20$ & $\mathrm{n}=16$ & $\mathrm{n}=14$ \\
\hline
\end{tabular}

Previously infected subjects $=$ subjects previously infected with SARS-CoV-2

Not previously infected subjects $=$ subjects not infected with SARS-CoV-2

$\mathrm{n}=$ number of subjects

median = median of anti - SARS-CoV-2 S titers

$\mathrm{IR}=$ Interquartile Range of anti - SARS-CoV-2 S titers

For previously infected subjects: T0 $=$ baseline pre-vaccination after SARS-CoV-2 infection, $\mathrm{T} 1=8$ days after the unique dose, T2 I = 1 month after the unique dose, T3 = 3 months after the unique dose, T6 = 6 months after the unique dose, T9 $=9$ months after the unique dose, $\mathrm{T}$ boost $=8$ days after the administration of a further boost dose. For not infected subjects: $\mathrm{T} 0=$ baseline pre-vaccination, $\mathrm{T} 1=20$ days after the first dose, T2 I = 8 days after the second dose, T2 II = 1 month after the second dose, T3 = 3 months after the second dose, T6 $=6$ months after the second dose, $\mathrm{T} 9=9$ months after the second dose, $\mathrm{T}$ boost $=8$ days after the administration of boost dose.

Table 4 Antibody titers' fold changes (FC) in previously infected and not previously infected cohorts of healthcare providers to monitor vaccine immune responses 
Analyzed cohorts

mean FC $\min \mathrm{FC} \quad \max \mathrm{FC}$

\section{Previously infected subjects}

\begin{tabular}{|llll}
\hline T1 - T0 & 2.0 & 1.5 & 1.9 \\
\hline T2 - T1 & 0.1 & -0.1 & 0.2 \\
\hline T2 - T0 & 2.1 & 1.5 & 3.1 \\
\hline T3 - T2 & -0.6 & -0.6 & -0.5 \\
\hline T6 - T3 & -0.2 & -0.2 & -0.2 \\
\hline T9 - T6 & -0.2 & -0.2 & -0.1 \\
\hline T boost - T6 & 1.5 & 1.5 & 1.5 \\
\hline T boost - T9 & 1.7 & 1.7 & 1.7 \\
\hline T boost - T2 & 0.7 & 0.7 & 0.8 \\
\hline T boost - T0 & 2.3 & 2.3 & 2.4
\end{tabular}

Not previously infected subjects

\begin{tabular}{lccc}
\hline T1 - T0 & 1.6 & 0.6 & 2.4 \\
\hline T2 I - T1 & 2.0 & 1.3 & 3.0 \\
\hline T2 II - T2 I & -0.4 & -0.7 & -0.2 \\
\hline T2 I - T0 & -0.3 & -0.5 & -0.1 \\
\hline T3 - T2 II & -0.2 & -0.5 & 0.1 \\
\hline T6 - T3 & -0.1 & -0.3 & 0.0 \\
\hline T9 - T6 & -0.1 & -0.3 & 0.0 \\
\hline T boost - T6 & 1.7 & 1.2 & 2.6 \\
\hline T boost - T9 & 1.9 & 1.3 & 2.6 \\
\hline T boost - T2 I & 0.9 & 0.4 & 1.3 \\
\hline T boost - T2 II & 1.2 & 0.7 & 1.8 \\
\hline T boost - T0 & 4.5 & 4.1 & 4.9
\end{tabular}


Previously infected subjects $=$ subjects previously infected with SARS-CoV-2

Not previously infected subjects $=$ subjects not infected with SARS-CoV-2

For previously infected subjects: T0 $=$ baseline pre-vaccination after SARS-CoV-2 infection, $\mathrm{T} 1=8$ days after the unique dose, T2 I = 1 month after the unique dose, T3 = 3 months after the unique dose, T6 = 6 months after the unique dose, T9 $=9$ months after the unique dose, $\mathrm{T}$ boost $=8$ days after the administration of a further boost dose. For not infected subjects: $\mathrm{T} 0=$ baseline pre-vaccination, $\mathrm{T} 1=20$ days after the first dose, T2 I = 8 days after the second dose, T2 II = 1 month after the second dose, T3 = 3 months after the second dose, T6 = 6 months after the second dose, T9 = 9 months after the second dose, $\mathrm{T}$ boost $=8$ days after the administration of boost dose.

mean FC = mean fold changes of anti - SARS-CoV-2 S mean geometric titers

min $\mathrm{FC}=$ minimum value of fold changes of anti $-\mathrm{SARS}-\mathrm{CoV}-2 \mathrm{~S}$ mean geometric titers $\max \mathrm{FC}=$ maximum value of fold changes of anti $-\mathrm{SARS}-\mathrm{CoV}-2 \mathrm{~S}$ mean geometric titers $\mathrm{n}=$ number of subjects

\section{Figures}




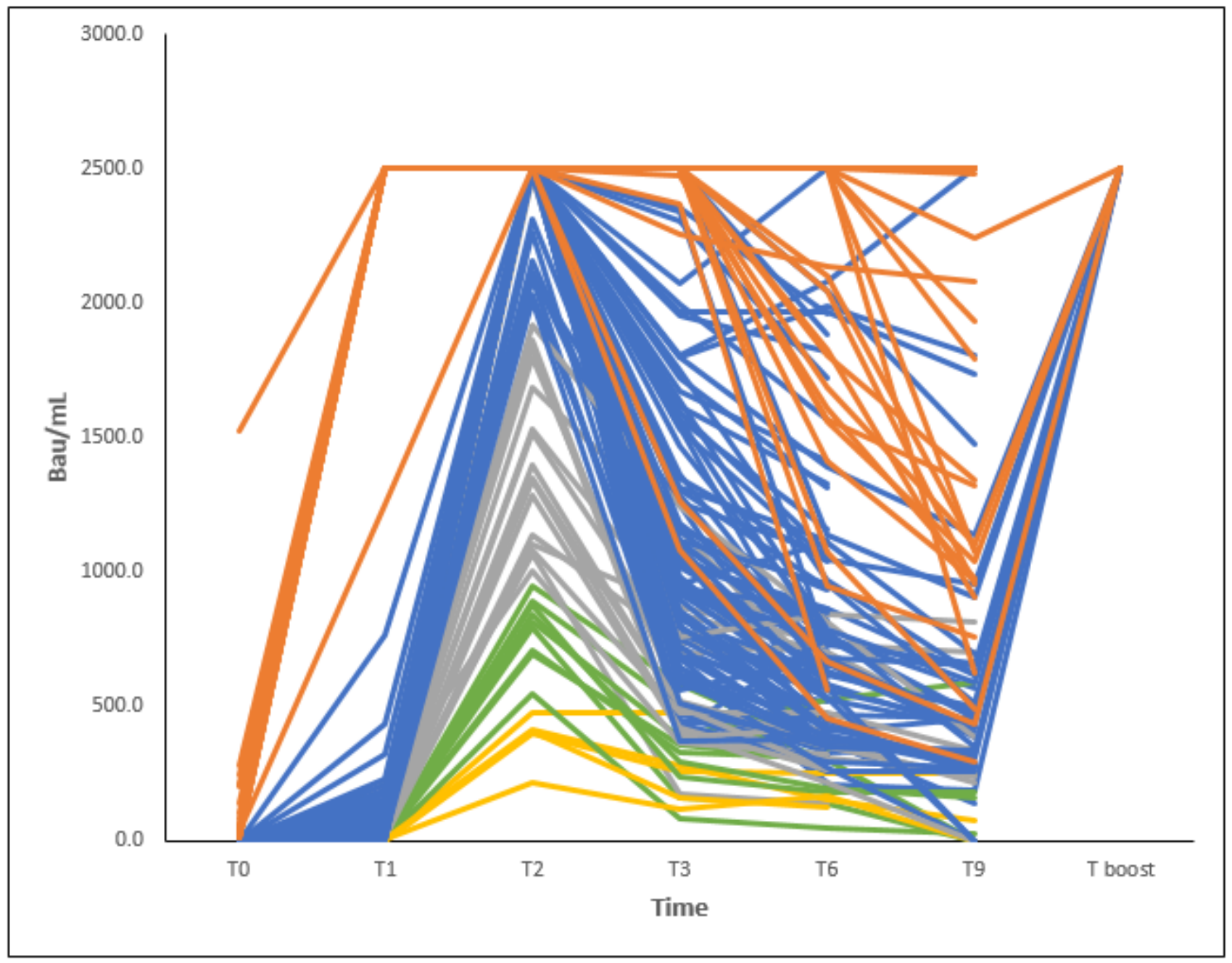

\section{Figure 1}

Vaccine immune response monitoring in previously infected and not previously infected cohorts of healthcare providers

For not infected subjects: $\mathrm{T} 0$ = baseline pre-vaccination, $\mathrm{T} 1=20$ days after the first dose, $\mathrm{T} 2=8$ days after the second dose, T3 $=3$ months after the second dose, T6 $=6$ months after the second dose, T9 $=9$ months after the second dose, $\mathrm{T}$ boost $=8$ days after the administration of boost dose. For previously infected subjects: T0 = baseline pre-vaccination after SARS-CoV-2 infection, T1 $=8$ days after the unique dose, $\mathrm{T} 2=1$ month after the unique dose, T3 $=3$ months after the unique dose, $\mathrm{T} 6=6$ months after the unique dose, T9 $=9$ months after the unique dose, $\mathrm{T}$ boost $=8$ days after the administration of a further boost dose. Orange lines: subjects previously infected with SARS-CoV-2. Yellow lines: subjects not previously infected and with T2 titers $<500.0 \mathrm{BAU} / \mathrm{mL}$. Green lines: subjects not previously infected and with T2 titers $<1000.0 \mathrm{BAU} / \mathrm{mL}$ and $\geq 500.0 \mathrm{BAU} / \mathrm{mL}$. Grey lines: subjects not previously infected and with T2 titers $<2000.0 \mathrm{BAU} / \mathrm{mL}$ and $\geq 1000.0 \mathrm{BAU} / \mathrm{mL}$. Blue lines: subjects not previously infected and with $\mathrm{T} 2$ titers $\geq 2000.0 \mathrm{BAU} / \mathrm{mL}$. 


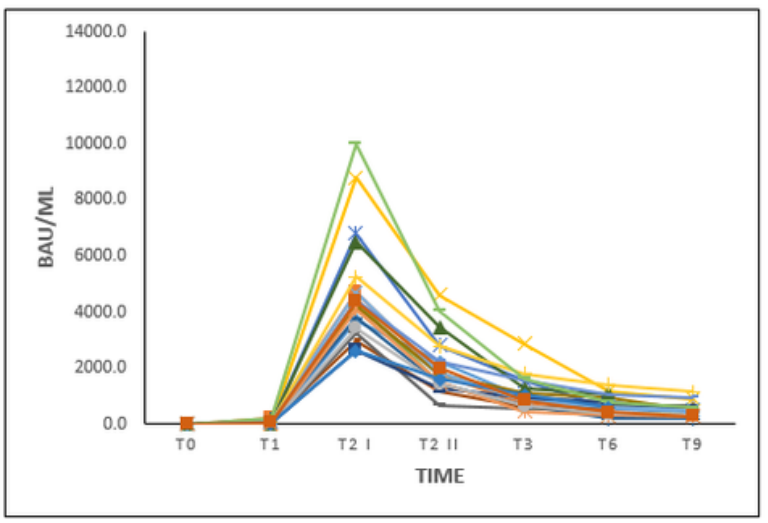

(a)

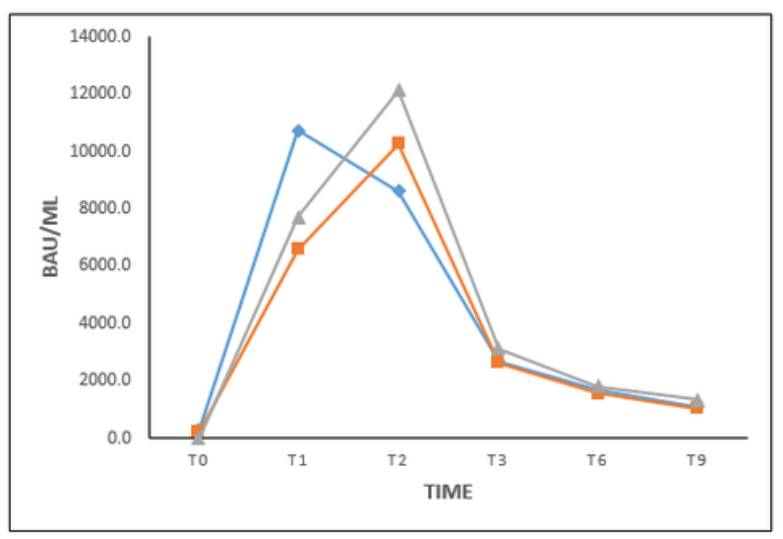

(b)

\section{Figure 2}

a Vaccine immune response decline in not previously infected healthcare providers' diluted samples

$\mathrm{T} 0$ = baseline pre-vaccination, $\mathrm{T} 1=20$ days after the first dose, $\mathrm{T} 2 \mathrm{I}$ I 8 days after the second dose, T2 II $=1$ month after the second dose, T3 $=3$ months after the second dose, T6 = 6 months after the second dose, T9 $=9$ months after the second dose. Each colored line represents a subject.

b Vaccine immune response decline in previously infected healthcare providers' diluted samples

T0 = baseline pre-vaccination after SARS-CoV-2 infection, T1 = 8 days after the unique dose, T2 = 1 month after the unique dose, T3 $=3$ months after the unique dose, T6 $=6$ months after the unique dose, T9 $=9$ months after the unique dose. Each colored line represents a subject.

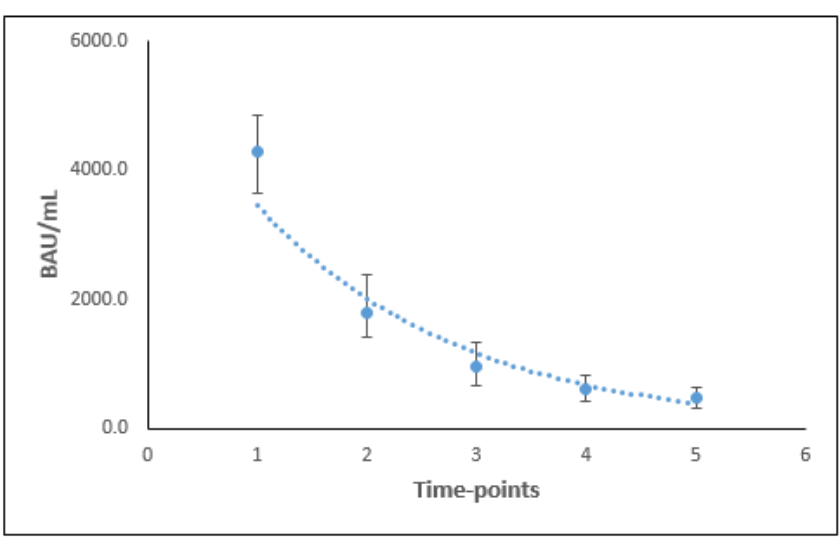

(a)

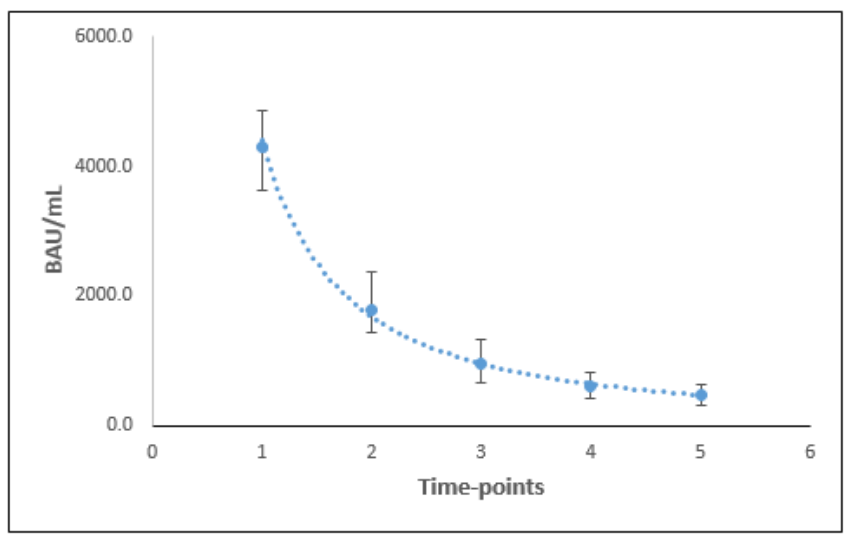

(b) 


\section{Figure 3}

a Exponential model to study and calculate the antibody decline kinetics in not previously infected subjects

Different time-points: $1=\mathrm{T} 2 \mathrm{I}, 2=\mathrm{T} 2 \mathrm{II}, 3=\mathrm{T} 3,4=\mathrm{T} 6,5=\mathrm{T} 9$. Blue dot line indicates the graphic representation of the exponential model, the single blue dots the median values at different time-points and black vertical lines the interquartile range of distributions.

b Power function model to study and calculate the antibody decline kinetics in not previously infected subjects

Different time-points: $1=\mathrm{T} 2 \mathrm{I}, 2=\mathrm{T} 2 \mathrm{II}, 3=\mathrm{T} 3,4=\mathrm{T} 6,5=\mathrm{T} 9$. Blue dot line indicates the graphic representation of the power function model, the single blue dots the median values at different timepoints and black vertical lines the interquartile range of distributions.

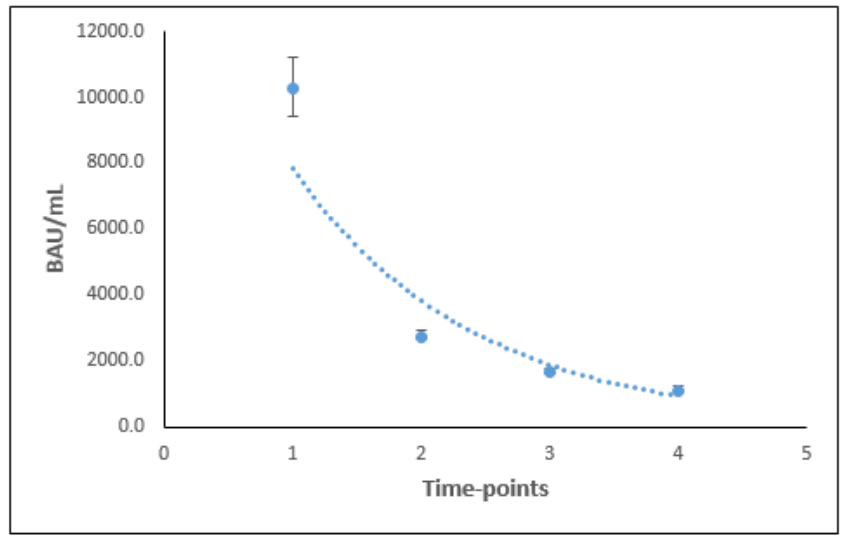

(a)

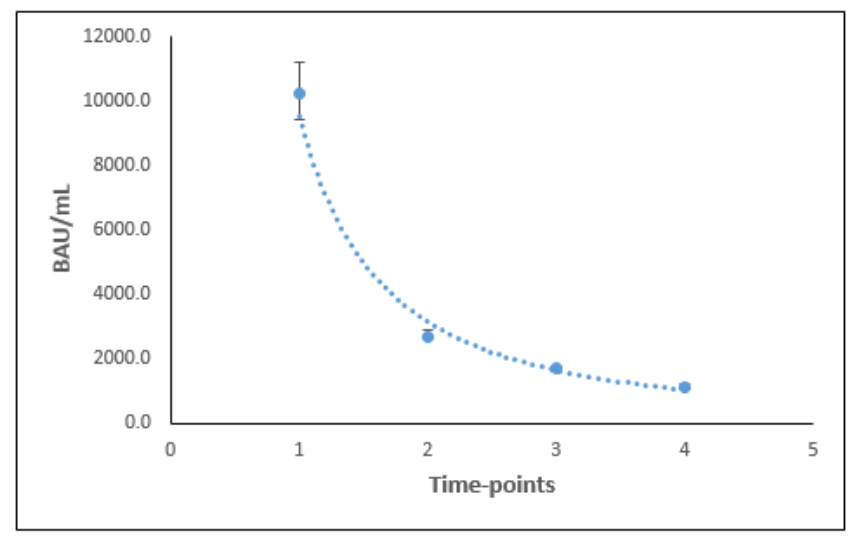

(b)

\section{Figure 4}

a Exponential model to study and calculate the antibody decline kinetics in previously infected subjects

Different time-points: $1=\mathrm{T} 2,2=\mathrm{T} 3,3=\mathrm{T} 6,4=\mathrm{T}$. . Blue dot line indicates the graphic representation of the exponential model, the single blue dots the median values at different time-points and black vertical lines the interquartile range of distributions.

b Power function model to study and calculate the antibody decline kinetics in previously infected subjects 
Different time-points: $1=\mathrm{T} 2,2=\mathrm{T} 3,3=\mathrm{T} 6,4=\mathrm{T}$. . Blue dot line indicates the graphic representation of the power function model, the single blue dots the median values at different time-points and black vertical lines the interquartile range of distributions.

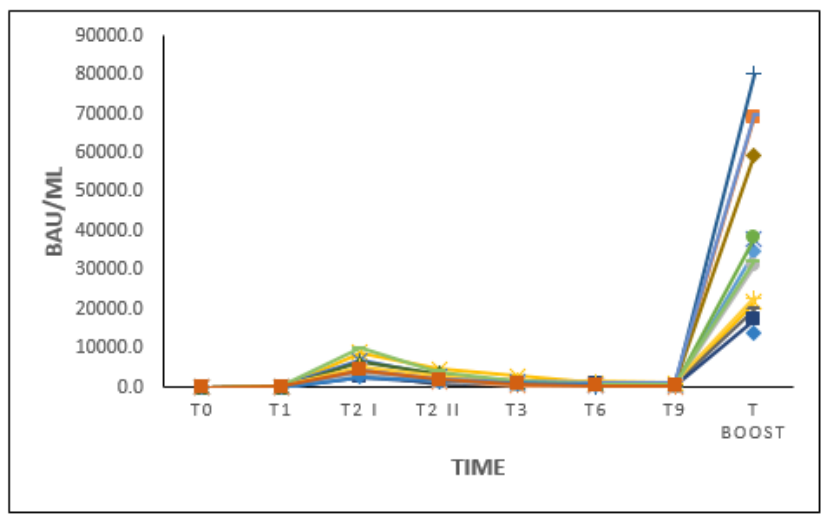

(a)

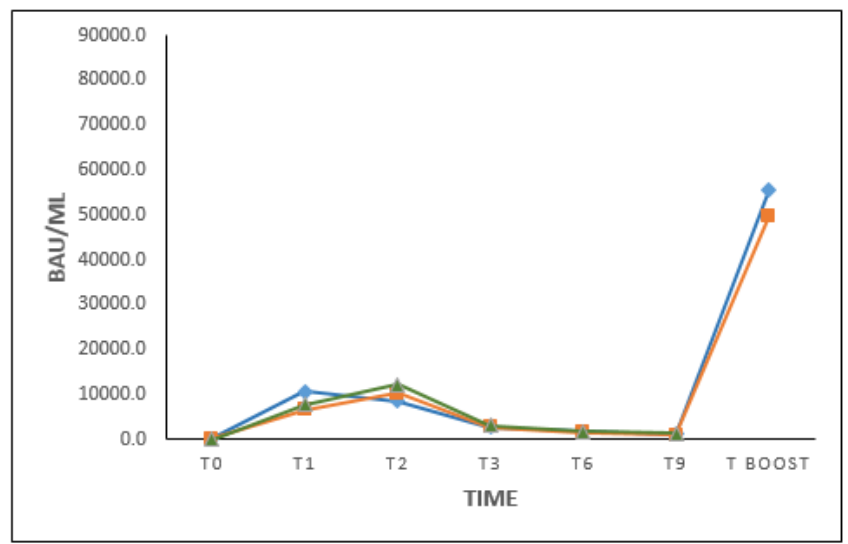

(b)

\section{Figure 5}

a Vaccine immune response increase after boost dose administration in not previously infected healthcare providers' diluted samples

T0 = baseline pre-vaccination, $\mathrm{T} 1$ = 20 days after the first dose, T2 I = 8 days after the second dose, T2 II $=1$ month after the second dose, $\mathrm{T} 3=3$ months after the second dose, T6 $=6$ months after the second dose, $\mathrm{T} 9=9$ months after the second dose, $\mathrm{T}$ boost $=8$ days after the administration of a further boost dose. Each colored line represents a subject.

b Vaccine immune response increase after boost dose administration in previously infected healthcare providers' diluted samples

T0 = baseline pre-vaccination after SARS-CoV-2 infection, T1 = 8 days after the unique dose, T2 = 1 month after the unique dose, T3 = 3 months after the unique dose, T6 = 6 months after the unique dose, $\mathrm{T} 9=9$ months after the unique dose, $\mathrm{T}$ boost $=8$ days after the administration of a further boost dose. Each colored line represents a subject. 